\title{
Agrp Neurons: Segregated Feeding Regulation through Different Projections \\ Qingchun Tong*
}

The Brown Foundation Institute of Molecular Medicine, The University of Texas Health Science Center at Houston, USA

Keywords: AgRP neurons; Parabrachial nucleus; Paraventricular hypothalamus; Feeding

Bioenergetics is a subject concerned with the energy involved in making and breaking of chemical bonds in the molecules found in biological organisms. Energy transformation is fundamental to growth, development and metabolism of biological organisms. The ability to harness energy from a variety of metabolic pathways is a property of all living organisms. For a multi-organ system like rodents and mammals, different organs have been evolved to possess distinct functions to maintain a balanced energy transformation. For example, the brain controls the intake of energy (mostly carbohydrates, fats, and proteins), which is absorbed by the digestive system, assorted by the liver and distributed to the body by the circulation system. A disturbance in balance between energy intake and energy necessarily required for body usage will lead to changes in metabolism and body growth (body weight). During evolution when food is not always available, excess energy is stored as fat, which can be used when food is scarce. Thus, for a given living subject, energy balance is depicted as energy intake=internal heat produced+external work+energy storage (fat). An increased body weight due to excessive fat accumulation is defined as obesity.

The current obesity epidemic and lack of efficient therapeutics demand a clear understanding of the mechanism underlying body weight regulation. It is now well established that the brain, especially the hypothalamus, maintains body weight homeostasis by effectively adjusting food intake and energy expenditure (internal heat production and external work) in response to changes in levels of various nutritional status indicators such as leptin, an adipose tissue derived hormone. In regard to feeding control, emerging results demonstrate an essential role for agouti-related peptide (AgRP) neurons, which is located in the arcuate nucleus (Arc) of the hypothalamus. AgRP neurons and proopiomelanocortin (POMC) neurons, also located in the Arc, are components of the melanocortin system, a well established neurocuit important for energy balance regulation. Indeed, direct leptin action on AgRP neurons has been revealed by the obesity phenotypes caused by specific deletion of LepR in AgRP neurons [1]. AgRP neurons directly sense changes in nutritional status hormones such as leptin, insulin and others to modulate energy homeostasis. As a result, AgRP neuron activity dramatically increases in response to fasting, signaling a need to eat [2]. Neuropeptide Y (NPY) and AgRP, two neuropeptides released from AgRP neurons, potently induce feeding, consistent with the role of AgRP neurons in promoting feeding [3]. Interestingly, compared to controls, increasing the activity level of AgRP neurons induces hyperphagia whereas reducing the activity level of those neurons induces hypophagia [4], suggesting that the activity level of AgRP neurons correlates with the level of feeding (Figure 1). These data suggest that AgRP neurons are both sufficient and required for feeding regulation. Notably, recent two studies identified two distinct projections from AgRP neurons, which mediate segregated roles of AgRP neurons in feeding.

In addition to NPY and AgRP, AgRP neurons also release GABA, which is required for AgRP neurons in the regulation of energy balance [5]. Interestingly, specific lesion of AgRP neurons in neonates produces a grossly normal phenotype while that in adult mice leads to starvation to death [6]. Importantly, $\mathrm{Wu}$ and colleagues found that the starvation phenotype can be rescued by specific delivery of GABA-A receptor agonist in the parabrachial nucleus (PBN) [7], highlighting an important role for GABAergic projections from AgRP neurons to PBN. Consistently, adult lesion of AgRP neurons increases PBN neuron activity. Taken together, these data suggest a role for a decreased GABAergic tone from AgRP neurons to PBN in modulating hypophagia response. Notably, although adult lesion of AgRP neurons also increases the activity of paraventricular hypothalamic (PVH) neurons, as demonstrated by strong C-Fos expression in response to AgRP neuron lesion, the starvation phenotype cannot be rescued by inhibiting PVH neuron activities $[7,8]$. This contrasting data argue that the regulation of hypophagia by AgRP neurons is selectively mediated by AgRP neurons $\rightarrow$ PBN projections. An interesting question is that whether this projection also mediates hyperphagic effects produced by increased AgRP neuron activity. Surprisingly, Atasoy and colleagues found that the activation of PBN fails to reverse the hyperphagic effects

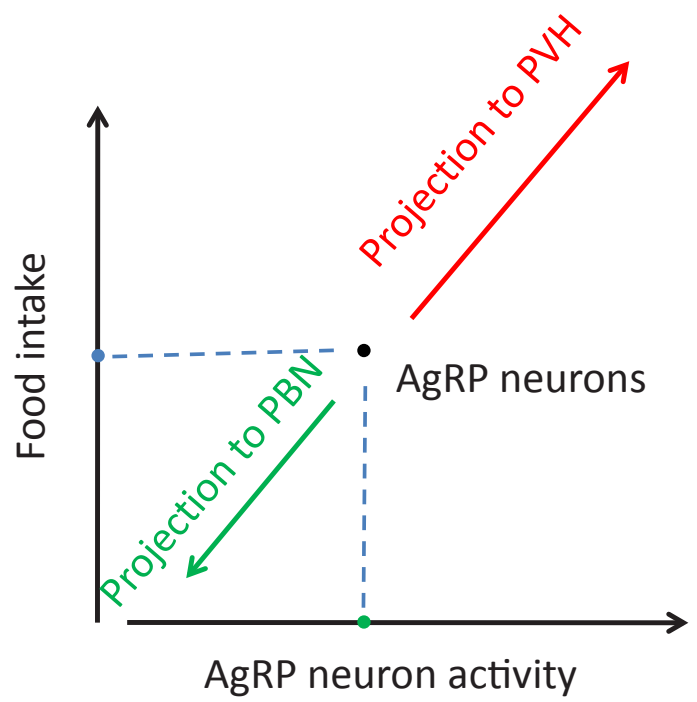

Figure 1: A simplified model illustrating a correlation between AgRP neuron activity and food intake. $\bullet$ : presumed AgRP neuron activity level in normal physiologic state; $\bullet$ : presumed feeding levels in normal physiologic state.

*Corresponding author: Qingchun Tong, The Brown Foundation Institute of Molecular Medicine, The University of Texas Health Science Center at Houston, 1825 Pressler Street, Houston, Texas, 77030, USA, E-mail: qingchun.tong@uth.tmc.edu

Received August 24, 2012; Accepted August 27, 2012; Published August 29, 2012

Citation: Tong Q (2012) Agrp Neurons: Segregated Feeding Regulation through Different Projections. Bioenergetics 1:e109. doi:10.4172/2167-7662.1000e109

Copyright: (C) 2012 Tong Q. This is an open-access article distributed under the terms of the Creative Commons Attribution License, which permits unrestricted use, distribution, and reproduction in any medium, provided the original author and source are credited. 
Citation: Tong Q (2012) Agrp Neurons: Segregated Feeding Regulation through Different Projections. Bioenergetics 1:e109. doi:10.4172/2167$7662.1000 \mathrm{e} 109$

Page 2 of 2

by AgRP neuron activation [9], suggesting that AgRP neuron induced hyperphagia is not due to a heightened GABAergic tone to PBN. In contrast, they found that the activation of PVH neurons reverses the hyperphagic effects by AgRP neurons activation [9], suggesting that AgRP neuron induced hyperphaga is due to heightened GABAergic tone to the PVH. Taken together, as shown in (Figure 1), these data suggest that segregated roles of two major AgRP neuron GABAergic projections: one to $\mathrm{PBN}$ selectively mediating hypophagia effects produced by reduced AgRP neuron activity and the other to the PVH selectively mediating hyperphagia effects produced by enhanced AgRP neuron activity.

$\mathrm{Wu}$ and colleagues used a combination of mouse genetics and pharmacology to achieve specific lesion of adult AgRP neurons. Atasoy and colleagues employed the state-of-the-art optogenetics and designer receptor exclusively activated by designer drugs (DREADD) to achieve specific activation of adult AgRP neurons. Given the complexity of feeding behavior, these studies represent a significant leap in understanding brain feeding circuits. It appears that AgRP neurons have been evolved to be one of major determinants for the individual to harness energy from the environment. With this in mind, modulating the activity of AgRP neurons represents one major strategy to control appetite for the current obesity epidemic [10].

It is certain that AgRP neurons are not the only key neurons in feeding control because lesion of the Arc including AgRP neurons leads to hyperphagia and obesity. Thus, in the Arc, there must be other food intake-inhibiting neurons, lesion of which overrides the action of AgRP neuron lesion. Nonetheless, based on the exciting results from AgRP neurons and using the newly developed techniques (mouse genetics, optogenetis and DREADD), future studies will provide more insights on brain circuits controlling food intake.

\section{Acknowledgement}

The research in the Tong lab is currently supported by a Scientist Development Award from the American Heart Association (10SDG3280017), National Institute of Health (1R01DK092605) and the Juvenile Diabetes Research Foundation.

\section{References}

1. van de Wall E, Leshan R, Xu AW, Balthasar N, Coppari R, et al. (2008) Collective and individual functions of leptin receptor modulated neurons controlling metabolism and ingestion. Endocrinology 149: 1773-1785.

2. Takahashi KA, Cone RD (2005) Fasting induces a large, leptin-dependent increase in the intrinsic action potential frequency of orexigenic arcuate nucleus neuropeptide Y/Agouti-related protein neurons. Endocrinology 146: 1043-1047.

3. Elmquist JK, Coppari R, Balthasar N, Ichinose M, Lowell BB (2005) Identifying hypothalamic pathways controlling food intake, body weight, and glucose homeostasis. J Comp Neurol 493: 63-71.

4. Krashes MJ, Koda S, Ye C, Rogan SC, Adams AC, et al. (2011) Rapid, reversible activation of AgRP neurons drives feeding behavior in mice. J Clin Invest 121: 1424-1428.

5. Tong Q, Ye CP, Jones JE, Elmquist JK, Lowell BB (2008) Synaptic release of GABA by AgRP neurons is required for normal regulation of energy balance. Nat Neurosci 11: 998-1000.

6. Luquet S, Perez FA, Hnasko TS, Palmiter RD (2005) NPY/AgRP neurons are essential for feeding in adult mice but can be ablated in neonates. Science 310: 683-685.

7. Wu Q, Boyle MP, Palmiter RD (2009) Loss of GABAergic signaling by AgRP neurons to the parabrachial nucleus leads to starvation. Cell 137: 1225-1234.

8. Wu Q, Howell MP, Cowley MA, Palmiter RD (2008) Starvation after AgRP neuron ablation is independent of melanocortin signaling. Proc Natl Acad Sci U S A 105: 2687-2692

9. Atasoy D, Betley JN, Su HH, Sternson SM (2012) Deconstruction of a neural circuit for hunger. Nature 488: 172-177.

10. Ren H, Orozco IJ, Su Y, Suyama S, Gutiérrez-Juárez R, et al. (2012) FoxO1 Target Gpr17 Activates AgRP Neurons to Regulate Food Intake. Cell 149: 1314-1326. 